\title{
Optimal Field Reconstruction of Distributed Process Systems from Partial Measurements
}

\author{
Míriam R. García, Carlos Vilas, Julio R. Banga \\ and Antonio A. Alonso* \\ Process Engineering Group, IIM-CSIC, Eduardo Cabello 6, 36208 Vigo, Spain.
}

May 11, 2012

\begin{abstract}
In this article, we develop a systematic approach for efficient field reconstruction in distributed process systems from a limited number of measurements. The approach generalizes previous methods for sensor placement so as to be able to handle field reconstruction problems in arbitrary spatial domains where complex nonlinear phenomena take place. Pattern formation in fluid dynamics or diffusion-reaction systems are examples exhibiting complex nonlinear distributed behaviours, specially when taking place in arbitrary $2 D$ or $3 D$ domains.

Our approach exploits the dissipative nature of diffusion-convection process and the underlying algebraic structure of the finite element method to efficiently construct field representations in terms of globally defined basis functions and to optimally select the placement of sensors. The results will be illustrated on a fluid dynamic process: the Rayleigh-Bénard problem.
\end{abstract}

Keywords: Distributed Process Systems, Monitoring and Control, Reduced Order Models, Field Reconstruction, Optimal Sensor Placement, Proper Orthogonal Decomposition (PODs).

${ }^{*}$ To whom correspondence should be addressed. Ph:+34 9862319 30, Fax: +34 986292762 ext: 251, email:antonio@iim.csic.es 


\section{Introduction}

Monitoring and control of chemical processes is at a high extent conditioned by the ability to efficiently reconstruct the current state of the process variables from a set of available measurements. The type and number of measurements needed will, in general, depend on the process under consideration, but it will always be guided by sensor reliability and cost as the critical parameters on which to support decisions in any control design project. Key questions (closely related with monitoring and control) include the possibility to infer a given process variable from others, much easier or cheaper to be measured, or the appropriate number of measurements (sensors) needed to produce a reliable reconstruction of the field.

In particular, such questions are specially relevant when dealing with processes variables exhibiting spatial distribution as in the case of many chemical reactors or processes with complex flow behavior.

Despite its long history, starting back in the $70^{\prime} s$, the problem of field reconstruction in distributed chemical processes is still attracting the attention of the process engineering community, specially with respect to nonlinear distributed phenomena and fluid dynamics ${ }^{1-3}$.

Early approaches to this problem ${ }^{4-6}$ made use of results from linear state space theory to develop dynamic observation schemes. These were based on the finite differences method (FDM) or finite element method (FEM) to approximate the original PDEs by a usually large set of ordinary differential equations (ODEs). On the other hand, measurements were placed so as to maintain the covariance matrix well conditioned. Different criteria were employed for that purpose, such as the maximization of the trace, or the determinant, associated to the covariance matrix. Recently, additional criteria were proposed including the maximization of measurement independence ${ }^{7}$ or maximization of the Grammian determinant ${ }^{8}$.

Although the need for systematic selection of optimal sensor location, as well as inputs and outputs pairings in distributed systems has become evident ${ }^{9}$, exhaustive search procedures which can be useful for placing a small number of sensors are still widely employed. Alternative approaches include the one proposed by Antoniades and Christofides ${ }^{10}$ to solve the placement problem by standard unconstrained optimization and taking advantage of the time scale separation properties of transport-reaction systems. The approach, although elegant, requires the process to be under control and restricts the number of sensors to the dimension of the slow dynamics. When dealing with a low number of inputs and outputs, optimal scheduling policies to place actuators and sensors have been recently proposed ${ }^{11}$. $\mathrm{In}^{2,12}$ the optimal sensor selection problem was formulated for a large number of sensors as that of minimizing the distance between the measurement subspace and the subspace which capture system dynamics. In these works, guided search algorithms were proposed.

The problem we deal with in this paper is that of efficient field reconstruction in arbitrary spatial domains where complex nonlinear phenomena take place. Pattern formation in fluid dynamics or diffusion-reaction systems are examples of systems exhibiting complex nonlinear distributed dynamics, specially when taking place in arbitrary $2 D$ or $3 D$ domains. In this context, capturing the essential dynamic features of the field from partial measurements usually calls for a large number of sensors (or sensor arrays) to be distributed over the spatial domain.

Seeking for the optimal sensor locations requires searching over extremely large dimensional search spaces, something which overrides any exhaustive search based approach and limits the efficiency of alternative guided search algorithms. On the other hand, field reconstruction from local basis such as those obtained by finite differences or finite elements requires the solution of a large number of ordinary differential or algebraic equations.

In order to overcome these limitations, the approach we follow makes use of the dissipative nature of linear or nonlinear diffusion-convection systems ${ }^{13-16}$ to produce reduced order approximations 
of the field. Such approximations are expressed in terms of globally defined basis functions such as those obtained by spectral methods or by the so-called Proper Orthogonal Decomposition (POD) $\operatorname{method}^{17,18}$.

In addition, we introduce the notion of subdomains of measurements as those which partition the whole spatial domain and are suitable for placing sensor arrays. We consider that each of these sensors measures the distribution of property in an arbitrary complex subdomain. For instance in a 2D spatial domain each sensor array takes photos at different times of a region with resolution (number of pixels) depending on the complexity of the phenomena taking place. In this way, the sensor placement problem is substituted by the problem of searching among regions (or subdomains) of measurements thus ensuring its solvability.

However, efficient computation of globally defined basis functions is highly conditioned by the spatial domain as it involves the solution of an eigenvalue problem associated with spatial operators such as the Laplacian or spatial integrals. For that purpose, we exploit the underlying structure of the finite element method to identify the algebraic equivalents of the corresponding infinite dimensional operators and thus compute the required basis. The algebraic structure given by FEM is also employed to formulate a generalized version of the sensor placement problem which now can be solved by guided search methods as the ones developed by Alonso et al. ${ }^{2,12}$.

Finally, it must be remarked that this approach, which combines low-dimensional spaces with FEM basis, can also be applied to other systematic selection criteria such as the trace or determinant of the Gramian ${ }^{7,8}$ or the covariance matrix obtained from statistical arguments ${ }^{19,20}$.

The paper is structured as follows: first and for the sake of completeness, the classical finite element method is briefly described in Section 2 as it provides the underlying algebraic structure on which the field will be expressed and the optimal sensor problem stated. In Section 3, lowdimensional approximations of the field and particularly the so-called POD method, are discussed in the context of the FEM. The methodology we propose to undertake the on-line field reconstruction problem, including optimal sensor placement is discussed in Section 4. Finally, in Section 5, the approach will be illustrated on the well known natural convection Rayleigh-Bénard Problem.

\section{Distributed transport-reaction processes in the Frame- work of the Finite Element Method.}

The class of systems we are dealing with are transport-reaction processes described by a set of quasi-linear partial differential equations (PDEs) of the form:

$$
\frac{\partial u}{\partial t}=\mathrm{L}(u)+\sigma(u)+\sigma^{o} \quad \forall \xi \in \Omega
$$

where $u$ represents the vector field which depends on time $t$ over the interval $[0,+\infty)$ and on spatial coordinates $\xi$. These are defined over the open spatial domain $\Omega \subset \mathbb{R}^{d}$ with smooth boundary $\partial \Omega$ and $d=1,2,3$ being the considered dimension $(1 D, 2 D$ or $3 D$ problem).

The spatial operator $\mathrm{L}(\cdot)$ is of parabolic type and has the following general representation:

$$
\mathrm{L}(\cdot)=\left(\sum_{j, i=1}^{d} \frac{\partial}{\partial \xi_{j}}\left(\alpha_{j i} \frac{\partial}{\partial \xi_{i}}\right)-\sum_{i=1}^{d} \beta_{i} \frac{\partial}{\partial \xi_{i}}\right)
$$

where $\alpha_{j i}$ and $\beta_{i}$ are coefficients that in general may be also space dependent.

Nonlinearities, such as those induced by chemical reaction, are accommodated into the system (1a) through the production term $\sigma(u)$ which is assumed to be Lipschitz continuous. Additional 
terms, representing for instance constant or time dependent forcing, could be included in $\sigma^{o}$. System's description is completed with the following general set of boundary conditions:

$$
\vec{n} \nabla u+q u=g \quad \forall \xi \in \partial \Omega
$$

where $\nabla$ stands for the usual gradient operator included in the expression (1b) and $\vec{n}$ is a unit vector pointing outwards the boundary $\partial \Omega$. Parameters $q$ and $g$ are used in (1c) to represent either first or second order boundary conditions. In this way, Neumann boundary conditions can be obtained by choosing $q=0$. Alternatively, Dirichlet boundary conditions can be attained by selecting $q$ large enough so that $\vec{n} \nabla u$ can be disregarded as compared with $q u$. The resulting field at the boundary then becomes of the form $g / q$.

Solutions for system (1a) with natural boundary conditions (1c), can be found in Sobolev spaces equipped with an inner product and norm of the form:

$$
\langle f, g\rangle_{\Omega}=\int_{\Omega} f(\xi) g(\xi) d \xi \quad\|f\|_{\Omega}=\langle f, f\rangle_{\Omega}^{1 / 2}
$$

where $f$ and $g$ are given functions over the spatial domain $\Omega$. Formally, these Sobolev spaces are defined as:

$$
\begin{gathered}
H^{0}(\Omega) \equiv L^{2}(\Omega)=\left\{f(\xi): \Omega \rightarrow \mathbb{R} \text { such as }\|f(\xi)\|_{\Omega}<\infty\right\} \\
H^{q}(\Omega)=\left\{f(\xi) \in H^{q-1}(\Omega): \frac{\partial f(\xi)}{\partial \xi_{\imath}} \in H^{q-1}(\Omega), 1 \leq \imath \leq d, \text { with } q=1,2 \ldots\right\}
\end{gathered}
$$

in such a way that, whenever the solution belongs to any of these spaces, the field can be expressed as a complete convergent infinite series expansion of the form ${ }^{21}$ :

$$
u(t, \xi)=\sum_{i=1}^{\infty} m_{i}(t) w_{i}(\xi)
$$

where the spatial dependent functions $\left\{w_{\mathbf{i}}(\xi)\right\}$ form a basis of the solution space and $\left\{m_{i}(t)\right\}_{i=1}^{\infty}$ are the coordinates of the field with respect to such basis.

Furthermore, the variational or weak form of the solution of equation (1a) can be built upon functions $\left\{w_{\mathbf{i}}(\xi)\right\}$ which, satisfying the natural boundary conditions (1c), constitute a basis for the $H^{1}(\Omega)$ Sobolev space. In terms of the inner product (2), the variational form is built by projecting the PDE set over the given basis functions $\left\{w_{\mathbf{i}}(\xi)\right\}$ and making use of the divergence theorem to relax the second order derivatives.

In fact, the finite element method makes use of this approach and seeks for solutions $u(t, \xi)$ of (1a) and (1c) on the $H^{1}(\Omega)$ Sobolev space. For the sake of completeness and since this formulation will be employed along the paper, we next summarize the basic steps involved in the Finite Element Method. A complete discussion of this method can be found elsewhere (see for instance ${ }^{22}$ ).

- Selection of an appropriate finite space which approximates $\left\{w_{\mathbf{i}}(\xi)\right\} \in H^{1}(\Omega)$. Since the infinite expansion (4) is a Cauchy convergent series ${ }^{21}$, the field in $H^{1}(\Omega)$ can be approximated by a function space $X_{\mathbf{n}} \subset H^{1}(\Omega)$ with finite dimension $\mathbf{n}$, so that:

$$
u(t, \xi) \simeq \widetilde{u}(t, \xi)=\sum_{\mathbf{i}=1}^{\mathbf{n}} \mathrm{u}_{\mathbf{i}}(t) \psi_{\mathbf{i}}(\xi)
$$

where $\left\{\psi_{\mathbf{i}}\right\}_{\mathbf{i}=1}^{\mathbf{n}}$ represents a basis for the subspace $X_{\mathbf{n}}$, and $\left(\mathrm{u}_{1}, \mathrm{u}_{2}, \ldots, \mathrm{u}_{\mathbf{n}}\right)$ are the coordinates with respect to such basis. 
- Construction of the finite element basis. The closed domain $\bar{\Omega}=\Omega \cup \partial \Omega$ is split up into $n_{e}$ triangulations $\tau_{\mathbf{j}}\left(\bar{\Omega}=\cup_{\mathbf{j}=1}^{n_{e}} \tau_{\mathbf{j}}\right)$ (the finite elements) in such a way that each of them is closed with non empty interior and Lipschitz boundary. Moreover, the intersection between the interior of any pair of triangulations must be empty and the frontier of any triangulation either coincide with the boundary of an adjoint element or with the limit of the domain. In order to ensure continuity of the solution, the elements must be connected so that their nodes are located in at least, the $\mathbf{n}$ vertex of the triangulations, whose coordinates we denote by $\left\{b_{\mathbf{j}}\right\}_{\mathbf{j}=1}^{\mathbf{n}} \in \mathbb{R}^{d}$. In addition, each of the basis functions $\left\{\psi_{\mathbf{i}}(\xi)\right\}_{\mathbf{i}=1}^{\mathbf{n}}$ which generate the space $X_{\mathbf{n}}$ must satisfy that:

$$
\psi_{\mathbf{i}}\left(\left\{b_{\mathbf{j}}\right\}_{\mathbf{j}=1}^{\mathbf{n}}\right)=\left\{\psi_{\mathbf{i}}\left(b_{\mathbf{j}}\right)\right\}_{\mathbf{j}=1}^{\mathbf{n}} \quad \text { with } \quad \psi_{\mathbf{i}}\left(b_{\mathbf{j}}\right)=\delta_{\mathbf{i j}} \quad \forall \mathbf{i}, \mathbf{j}=\mathbf{1}, \ldots, \mathbf{n}
$$

where $\delta_{i j}$ stands for the Kronecker delta.

Note that these basis functions can be expressed as polynomials inside the finite elements. Thus, they can be built by assembling local basis defined over each finite element. Depending on the degree of the polynomial used to approximate the solution inside each element, additional nodes may be required inside the triangulations.

Combining (5) with the set of basis functions satisfying (6), it can be easily seen that the coordinates correspond with the value of the field at the nodes of the FEM mesh, i.e.:

$$
\widetilde{u}\left(t, b_{\mathbf{j}}\right) \simeq \sum_{\mathbf{i}=1}^{\mathbf{n}} \mathrm{u}_{\mathbf{i}}(t) \psi_{\mathbf{i}}\left(b_{\mathbf{j}}\right)=\mathrm{u}_{\mathbf{j}}(t) \quad \forall \mathbf{j}=\mathbf{1}, \ldots, \mathbf{n}
$$

In the same way, the discrete version of the field in the FEM mesh with coordinates $\left\{b_{\mathbf{j}}\right\}_{\mathbf{j}=1}^{\mathbf{n}}$ can be constructed by using relation (6) as:

$$
\widetilde{u}\left(t,\left\{b_{\mathbf{j}}\right\}_{\mathbf{j}=1}^{\mathbf{n}}\right) \simeq \sum_{\mathbf{i}=1}^{\mathbf{n}} \mathrm{u}_{\mathbf{i}}(t)\left\{\psi_{\mathbf{i}}\left(b_{\mathbf{j}}\right)\right\}_{\mathbf{j}=1}^{\mathbf{n}}
$$

so that $\widetilde{u}$ can be encoded on a vector of the form:

$$
U(t)=\left[\mathrm{u}_{\mathbf{1}}(t), \mathrm{u}_{\mathbf{2}}(t), \ldots, \mathrm{u}_{\mathbf{n}}(t)\right]^{T} \in \mathbb{R}^{\mathbf{n}}
$$

As shown $i^{22}$, the approximation error to the original field can be bounded as a function of the size (length) of the element and the degree of the polynomial employed. In this way, the accuracy of the approximation can be controlled (and thus improved) by increasing the polynomial degree and decreasing the length of the elements employed.

Finally, it must be pointed out that the projection of the original PDE (1a) onto the FEM basis produces a set of ODEs with the following structure:

$$
\mathcal{D} \mathcal{A} \frac{d U(t)}{d t}+(q \mathcal{Q}+\mathcal{C}+\mathcal{B E}) U(t)-\mathcal{F}^{u}=\mathcal{F}^{o}+\mathcal{G}
$$

with

$$
\begin{gathered}
\mathcal{D} \mathcal{A}_{\mathbf{i}, \mathbf{j}}=\int_{\Omega} \psi_{\mathbf{i}} \psi_{\mathbf{j}} d \xi \quad \mathcal{C}_{\mathbf{i}, \mathbf{j}}=\int_{\Omega} \nabla \psi_{\mathbf{i}} \nabla \psi_{\mathbf{j}} d \xi \quad \mathcal{B} \mathcal{E}_{\mathbf{i}, \mathbf{j}}=\int_{\Omega} \nabla \psi_{\mathbf{i}} \psi_{\mathbf{j}} d \xi \\
\mathcal{F}_{\mathbf{i}, \mathbf{j}}^{u}=\int_{\Omega} \sigma_{\mathbf{i}}^{u} \psi_{\mathbf{j}} d \xi \quad \mathcal{F}_{\mathbf{i}, \mathbf{j}}^{o}=\int_{\Omega} \sigma_{\mathbf{i}}^{o} \psi_{\mathbf{j}} d \xi \quad \mathcal{Q}_{\mathbf{i}, \mathbf{j}}=\int_{\partial \Omega} \psi_{\mathbf{i}} \psi_{\mathbf{j}} d \xi \quad \mathcal{G}_{\mathbf{i}, \mathbf{j}}=\int_{\partial \Omega} g_{\mathbf{i}} \psi_{\mathbf{j}} d \xi
\end{gathered}
$$

where now the states become the $U(t)$ coordinates thus resulting into a usually large dimensional ODE set, particularly when working in $2 D$ or $3 D$ domains. Nevertheless, the FEM structure we 
just described can be used to find operators to efficiently map the infinite dimensional space $H^{1}(\Omega)$ into its finite counterpart $X_{\mathbf{n}}$.

FEM equivalents of infinite dimensional operators such as domain integrals, gradients or Laplacians are summarized in Table 1 (see the Appendix A for more details) for arbitrary functions $f, g \in H^{1}(\Omega)$ approximated as in (8) so that $f(\xi) \rightarrow F \in \mathbb{R}^{\mathbf{n}}$ and $g(\xi) \rightarrow G \in \mathbb{R}^{\mathbf{n}}$. These operators will be employed in the next Sections to develop low dimensional approximations of the solution based on globally defined basis functions.

\begin{tabular}{|ccc|}
\hline Continous & & Discrete \\
\hline \hline $\int_{\Omega} h(\xi) f(\xi) d \xi$ & $\longrightarrow$ & $H^{T}(\mathcal{D} \mathcal{A}) F$ \\
$\int_{\Omega} h(\xi) \nabla f(\xi) d \xi$ & $\longrightarrow$ & $H^{T}(\mathcal{B E}) F$ \\
$\int_{\Omega} h(\xi) \Delta f(\xi) d \xi$ & $\longrightarrow$ & $H^{T}(\mathcal{G})-H^{T}(\mathcal{C}+q \mathcal{Q}) F$ \\
$\nabla f(\xi)$ & $\longrightarrow$ & $\left(\mathcal{D} \mathcal{A}^{-1}\right)(\mathcal{B E}) F$ \\
$\Delta f(\xi)$ & $\longrightarrow$ & $\left(\mathcal{D} \mathcal{A}^{-1}\right)(\mathcal{G})-\left(\mathcal{D} \mathcal{A}^{-1}\right)(\mathcal{C}+q \mathcal{Q}) F$ \\
\hline
\end{tabular}

Table 1: Algebraic relations to numerically compute integrals and derivatives using the FEM structure. $\triangle$ represents the Laplacian included in the parabolic operator (1b).

\section{Low dimensional approximations based on the FEM struc- ture}

Classical methods, such as FEM, need of high dimensional subspaces of $H^{1}(\Omega)$ to represent the field. As an alternative, the use of global spatial basis functions results in low dimensional subspaces which usually require a much smaller number of coordinates to approximate the solution.

Low dimensional approximations make use of relation (4) discussed in the previous Section, by replacing the basis function set $\left\{w_{i}(\xi)\right\}_{i=1}^{\infty}$ of the space $H^{1}(\Omega)$ by a globally defined set $\left\{\phi_{i}(\xi)\right\}_{i=1}^{\infty}$, and the time dependent functions $\left\{m_{i}(t)\right\}_{i=1}^{\infty}$ by the so-called modes set $\left\{c_{i}(t)\right\}_{i=1}^{\infty}$, so that the field can be formally re-written as:

$$
u(t, \xi)=\sum_{i=1}^{\infty} c_{i}(t) \phi_{i}(\xi)
$$

Each element $\phi_{i}(\xi)$ of the globally defined basis is computed as the solution of the following integral eigenvalue problem ${ }^{2,12,23}$ :

$$
\int_{\Omega} R\left(\xi, \xi^{\prime}\right) \phi_{i}\left(\xi^{\prime}\right) d \xi^{\prime}=\lambda_{i} \phi_{i}(\xi)
$$

where $\lambda_{i}$ corresponds with the eigenvalue associated with each global eigenfunction $\phi_{i}$. The kernel $R\left(\xi, \xi^{\prime}\right)$ in (12) is a symmetric and positive function of space, which, in accordance with Mercer's theorem, accepts the following expansion ${ }^{24}$ :

$$
R\left(\xi, \xi^{\prime}\right)=\sum_{i=1}^{\infty} \lambda_{i} \phi_{i}(\xi) \phi_{i}\left(\xi^{\prime}\right)
$$


Different basis sets can be obtained depending on the nature of the kernel $R\left(\xi, \xi^{\prime}\right)$, among which, the following have been widely considered (see for details ${ }^{2,12}$ ):

- Spectral decomposition ${ }^{21}$ : the kernel corresponds with the Green function associated with the Laplacian operator $\triangle$.

- Proper Orthogonal Decomposition (POD) or Karhunen-Loève expansion ${ }^{17}$ : the kernel corresponds with the two point spatial correlation function, defined as follows:

$$
R\left(\xi, \xi^{\prime}\right)=\lim _{T \rightarrow \infty} \frac{1}{T} \int_{0}^{T} u(\xi, t) u\left(\xi^{\prime}, t\right) d t
$$

where $u(\xi, t)$ represents the value of the field on the time interval $[0, T]$. Since usually only a finite number of snapshots of the field is available over the interval $[0, T]$, the kernel in (13) is approximated as:

$$
R\left(\xi, \xi^{\prime}\right)=\frac{1}{\ell} \sum_{j=1}^{\ell} u\left(\xi, t_{j}\right) u\left(\xi^{\prime}, t_{j}\right)
$$

$u\left(\xi, t_{j}\right)$ in $(14)$ corresponds with the value of the field at each instant $t_{j}$ and the summation extends over a sufficiently rich collection of uncorrelated snapshots at $j=1, . ., \ell^{17}$, which can be obtained either from experiments or by direct numerical simulation of the original PDE.

The dissipative nature of the chemical and the physical processes described by equation (1a) allows us to approximate the field by selecting a low-dimensional basis $\left\{\phi_{i}(\xi)\right\}_{i=1}^{m}$ which captures the most relevant features of the solution. Consequently the series (11) can be truncated by a usually small number of terms:

$$
u(t, \xi) \cong \sum_{i=1}^{m} c_{i}(t) \phi_{i}(\xi)
$$

As shown by Sirovich, Lumley and coworkers ${ }^{17,18}$ the POD approach produces a set of empirical basis functions optimal with respect to other possible expansions in the sense that, for a given number of functions, it captures most of the relevant dynamic behavior of the original distributed system in the range of initial conditions, parameters, inputs, and/or perturbations of the experimental data. In addition, the POD set can be easily computed for any distributed system regardless of the complexity of its domain or the class of nonlinearity. For these reasons, this method will be the one selected in this work to produce low dimensional approximations of the field.

The criterion chosen to truncate the expansion is closely related with the associated eigenspectrum $\left\{\lambda_{i}(\xi)\right\}_{i=1}^{\ell}$ as it is connected with the average distance of the data set to the subspace spanned by the PODs ${ }^{2}$ through the formula:

$$
D_{a v}^{2}=\left(\sum_{i=m+1}^{\ell} \lambda_{i}\right)
$$

which is also expressed as the percentage of the energy captured by the system ${ }^{17}$ :

$$
E(\%)=100 \times \frac{\sum_{i=1}^{m} \lambda_{i}}{\sum_{i=1}^{\ell} \lambda_{i}}
$$

In order to efficiently compute the globally defined eigenfunctions described above for arbitrarily complex spatial domains, we exploit the underlying algebraic FEM structure discussed in Section 2 and transform the integral eigenvalue problem (12) into an algebraic one. For that purpose, we 
make use of the relationships between the infinite dimensional operators (such as spatial derivatives or integrals) and their FEM algebraic counterparts presented in Appendix A and summarized in Table 1. In this way, combining (12) with (14) and rearranging terms, we have:

$$
\frac{1}{\ell} \sum_{j=1}^{\ell}\left(u\left(\xi, t_{j}\right) \int_{\Omega} u\left(\xi^{\prime}, t_{j}\right) \phi_{i}\left(\xi^{\prime}\right) d \xi^{\prime}\right)=\lambda_{i} \phi_{i}(\xi)
$$

Using the relations given in Table 1, the integral in (18) transforms into:

$$
\int_{\Omega} u\left(\xi^{\prime}, t_{j}\right) \phi_{i}\left(\xi^{\prime}\right) d \xi^{\prime} \rightarrow U^{T} \mathcal{D} \mathcal{A} \Phi_{i}
$$

where $U \in \mathbb{R}^{\mathbf{n}}$ and $\Phi_{i} \in \mathbb{R}^{\mathbf{n}}$ are the discrete versions of the field and the $i^{\text {th }}$ eigenfunction, respectively, obtained from (6) and (8). Applying the same relationships to the rest of terms in (18), the FEM counterpart of the original eigenvalue problem takes the form:

$$
\frac{1}{\ell} \sum_{j=1}^{\ell}\left[U\left(t_{j}\right) U\left(t_{j}\right)^{T}\right] \mathcal{D} \mathcal{A} \Phi_{i}=\lambda_{i} \Phi_{i}
$$

where the kernel (14) is now transformed into:

$$
R=\frac{1}{\ell} \sum_{j=1}^{\ell}\left[U\left(t_{j}\right) U\left(t_{j}\right)^{T}\right]
$$

Standard algebraic methods to solve eigenvalue problems ${ }^{25}$ can be now applied to (19) to obtain the corresponding POD set in the FEM domain. In this framework, the field (15) can be expressed in matrix form as:

$$
U\left(t_{s}\right)=\Phi C\left(t_{s}\right)
$$

where $U\left(t_{s}\right) \in \mathbb{R}^{\mathbf{n}}$ corresponds with the reconstructed field and $C\left(t_{s}\right)=\left[c_{1}\left(t_{s}\right), \ldots, c_{m}\left(t_{s}\right)\right]^{T} \in \mathbb{R}^{m}$ stands for the mode vector at each sampling time $t_{s}$. Matrix $\Phi\left(\left\{b_{\mathbf{j}}\right\}_{\mathbf{j}=1}^{\mathbf{n}}\right) \in \mathbb{R}^{\mathbf{n} \times m}$ collects in its columns the POD set elements obtained from (19) and ordered as:

$$
\Phi=\left[\Phi_{1}, \ldots, \Phi_{i}, \ldots, \Phi_{m}\right]=\left[\begin{array}{ccc}
\phi_{1}\left(b_{\mathbf{1}}\right) & \ldots & \phi_{m}\left(b_{\mathbf{1}}\right) \\
\vdots & \ddots & \vdots \\
\phi_{1}\left(b_{\mathbf{n}}\right) & \ldots & \phi_{m}\left(b_{\mathbf{n}}\right)
\end{array}\right]
$$

with $\left\{b_{\mathbf{i}}\right\}_{\mathbf{i}=1}^{\mathbf{n}}$ being the coordinates at the mesh nodes.

The method we just described to compute the PODs might be in some cases computationally involved as it requires working with large dimensional matrices. As an alternative to overcome the computational burden, the PODs can be obtained by the so-called indirect method, first proposed by Sirovich ${ }^{17,23}$. In this approach, the original eigenvalue problem (19) is transformed, by suitable algebraic manipulations, into the following equivalent but lower dimensional form:

$$
M \Theta_{i}=\lambda_{i} \Theta_{i} \quad \forall i=1, \ldots, m
$$

with

$$
M=\frac{1}{\ell}\left[\begin{array}{c}
U\left(t_{1}\right)^{T} \\
\vdots \\
U\left(t_{\ell}\right)^{T}
\end{array}\right] \mathcal{D} \mathcal{A}\left[U\left(t_{1}\right), \ldots, U\left(t_{\ell}\right)\right] \quad \Theta_{i}=\left[\begin{array}{c}
\theta_{1}^{i} \\
\vdots \\
\theta_{\ell}^{i}
\end{array}\right]
$$

The PODs are then reconstructed in terms of the $\left\{\Theta_{i}\right\}_{\mathbf{i}=1}^{m}$ eigenvectors and the original snapshots as:

$$
\Phi_{i}=\sum_{j=1}^{\ell} \theta_{j}^{i} U\left(t_{j}\right) \quad \forall i=1, \ldots, m
$$




\section{On-line field reconstruction in the reduced space}

In order to reconstruct the field $u(\xi, t)$ from a partial set of measurements, a collection of POD basis plus a mechanism for on-line estimation of the time dependent modes are required. As shown previously, PODs can be produced off-line from a representative set of snapshots obtained either from experiments or by direct numerical simulation. Estimation, on the other hand, can be obtained by means of dynamic observers such as those proposed by Alonso et al. ${ }^{2,12}$. These consist of a finite dimensional dynamic replica of the system obtained by projecting the original PDEs set on the reduced space spanned by the POD basis, with an extra-term which includes the partial measurements of the field. At this point it must be noted that the definition for observability employed is the usual one in finite dimensional systems, which applies not to the original PDE system but to the finite dimensional approximation which now has the formal structure of inputstate-output. When the observer is properly designed, asymptotic or even exponential convergence of the estimated modes to the true ones can be guaranteed. Alternatively, the static estimation of the modes at each measured time can be obtained without knowledge of the PDE set by means of the minimization of the distance between the available measurements and their associated field reconstruction. This is the class of approach we concentrate on, in the present work.

It must be pointed out, however, that in order to obtain any reliable on-line estimate of the modes from partial measurements, one needs to decide for a given set of sensors how many measurements are required and where these measurements need to be taken in the domain. This state information will be selected so to minimize the maximum angle between the low dimensions subspace and the space of measurements, and therefore, will be independent of the observer algorithm. With this state information we then reconstruct an approximation of the field which happens to be arbitrarily precise depending on the particular estimation and tuning procedures employed. In this Section, we concentrate on the optimal reconstruction problem by extending previous available results to arbitrary spatial domains.

\subsection{Reconstruction from subdomain measurements}

Let us consider the domain $\Omega$ decomposed into $N$, non-overlapping subdomains $\Omega_{j}$ so that $\Omega=$ $\cup_{j \in \mathcal{J}} \Omega_{j}$. $\mathcal{J}$ defines the set of natural numbers ordered from 1 to $N$, where each number can be associated to a sensor collecting values of the field in the subdomain $\Omega_{j}$. A graphical representation of the subdomains is presented in Figure 1. With these preliminaries, the reconstruction problem can be stated as follows:

For a given set of subdomains $\Omega_{\mathcal{S}}=\cup_{j \in \mathcal{S}} \Omega_{j} \subset \Omega$ with $\mathcal{S} \subset \mathcal{J}$ (i.e. a collection of elements belonging to $\mathcal{J})$ where the sensors are located, find the mode set $\left\{c_{i}(t)\right\}_{i=1}^{m}$ associated with the low-dimensional basis set $\left\{\phi_{\mathcal{S}, i}(\xi)\right\}_{i=1}^{m}$ (defined over $\Omega_{\mathcal{S}}$ ) which minimizes the distance between the measurements $y_{\mathcal{S}}$ and the estimates $\widehat{y}_{\mathcal{S}}$.

The distance between the data and the estimated set, will be measured in the norm $L^{2}\left(\Omega_{\mathcal{S}}\right)$, also known as the gappy norm by other authors ${ }^{1}$. This is equivalent to the summation extended over the measurement subdomain norms, so that:

$$
\|\varepsilon\|_{\Omega_{\mathcal{S}}}=\left(\int_{\Omega_{\mathcal{S}}}\left(y_{\mathcal{S}}-\widehat{y}_{\mathcal{S}}\right)^{2} d \xi\right)^{1 / 2}=\sum_{j \in \mathcal{S}}\left(\int_{\Omega_{j}}\left(y_{j}-\widehat{y}_{j}\right)^{2} d \xi\right)^{1 / 2}
$$

Employing such norm, mode estimation (and thus field reconstruction) reduces to the solution of the following optimization problem:

$$
\min _{\left\{\widehat{c}_{i}\left(t_{s}\right)\right\}_{i=1}^{m}} \frac{1}{2}\|\varepsilon\|_{\Omega_{\mathcal{S}}}^{2}=\min _{\left\{\widehat{c}_{i}\left(t_{s}\right)\right\}_{i=1}^{m}} \frac{1}{2} \int_{\Omega_{\mathcal{S}}}\left(y_{\mathcal{S}}\left(\xi, t_{s}\right)-\sum_{i=1}^{m} c_{i}\left(t_{s}\right) \phi_{\mathcal{S}, i}(\xi)\right)^{2} d \xi
$$




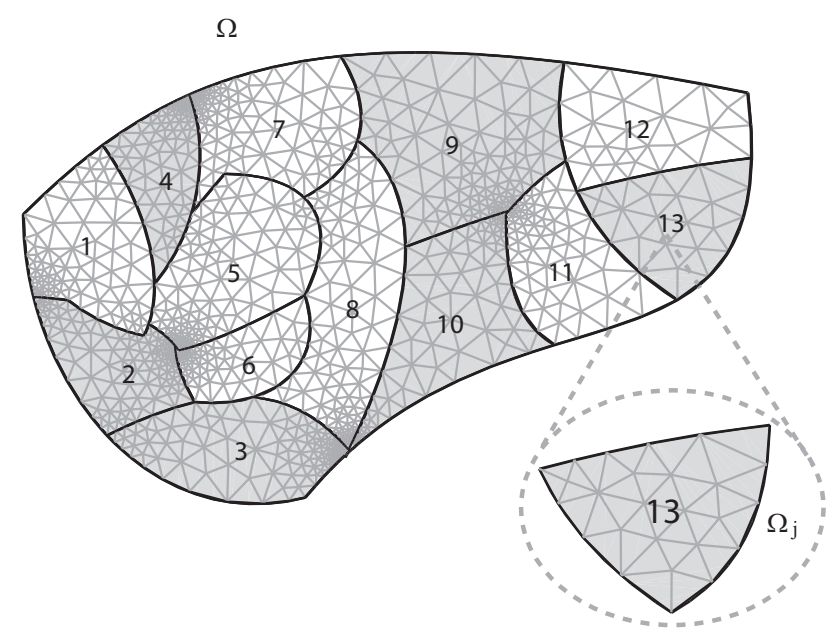

Figure 1: The spatial subdomain $\Omega$ is decomposed into 13 non-overlapping subdomains $\Omega_{j}$ with $\mathcal{J}=$ $(1, \ldots, 13)$. Highlighted in grey is a possible set of measurement subdomains $\mathcal{S}=(2,3,4,9,10,13)$

where $y_{\mathcal{S}}\left(\xi, t_{s}\right)$ and $\left\{\phi_{\mathcal{S}, i}(\xi)\right\}_{i=1}^{m}$ are the measurements and the basis functions associated with the domain $\Omega_{\mathcal{S}}$, respectively.

As discussed in the previous Section, the continuous optimization problem (23) can be transformed (by using the operators defined in Table 1) into the following discrete counterpart:

$$
\min _{\widehat{C} \in \mathbb{R}^{m}} J(\widehat{C})=\frac{1}{2} \sum_{j \in \mathcal{S}}\left(Y_{j}-\Phi_{j} \widehat{C}\right)^{T} \mathcal{D} \mathcal{A}_{j}\left(Y_{j}-\Phi_{j} \widehat{C}\right)
$$

where $\widehat{C}=\left[\widehat{c}_{1}\left(t_{s}\right), \ldots, \widehat{c}_{m}\left(t_{s}\right)\right]^{T} \in \mathbb{R}^{m}$ corresponds with the vector of estimated modes, $Y_{j}=$ $\left[y_{1}, \ldots, y_{n_{j}}\right]^{T} \in \mathbb{R}^{n_{j}}$ is the vector field measured over the $\Omega_{j}$ mesh $\left(n_{j}\right.$ denotes the number of pointwise measurements on such subdomain) with $j \in \mathcal{S}$, and the matrix $\Phi_{j} \in \mathbb{R}^{n_{j} \times m}$ collects the POD set over the same FEM mesh. Note that the gappy norm (22) can be evaluated by using matrices defined either over the whole domain $\Omega_{\mathcal{S}}$ or over the summation of the subdomains $\Omega_{j}$ for $j \in \mathcal{S}$ as in (22). The solution of problem (24) leads to:

$$
\widehat{C}=\Pi^{-1} \sum_{j \in \mathcal{S}} \Phi_{j}^{T} \mathcal{D} \mathcal{A}_{j} Y_{j} \quad \text { with } \quad \Pi=\sum_{j \in \mathcal{S}} \Phi_{j}^{T} \mathcal{D} \mathcal{A}_{j} \Phi_{j}
$$

Since each matrix $\mathcal{D} \mathcal{A}_{j}$ is symmetric and positive definite, it can be factorized as:

$$
\mathcal{D} \mathcal{A}_{j}=W_{j} \Lambda_{j} W_{j}^{T}
$$

where $W_{j}$ contains, as columns, the eigenvectors of $\mathcal{D} \mathcal{A}_{j}$ and $\Lambda_{j}$ is a diagonal matrix with the associated eigenvalues. This observation allows us to re-write matrix $\Pi \in \mathbb{R}^{m \times m}$ in (25) as follows:

$$
\begin{gathered}
\Pi=\sum_{j \in \mathcal{S}} \mathcal{Z}_{j}^{T} \mathcal{Z}_{j}=\mathcal{Z}_{\mathcal{S}}^{T} \mathcal{Z}_{\mathcal{S}} \\
\text { with } \\
\mathcal{Z}_{j}=\left(\Lambda_{j}^{1 / 2} W_{j}^{T} \Phi_{j}\right) \in \mathbb{R}^{n_{j} \times m} \quad \forall j \in \mathcal{S} \\
\mathcal{Z}_{\mathcal{S}}=\left[\mathcal{Z}_{\mathcal{S}(1)}^{T}, \ldots, \mathcal{Z}_{\mathcal{S}(j)}^{T}, \ldots, \mathcal{Z}_{\mathcal{S}\left(N_{\mathcal{S}}\right)}^{T}\right]^{T} \in \mathbb{R}^{n_{\mathcal{S}} \times m}
\end{gathered}
$$

where $N_{\mathcal{S}}$ and $n_{\mathcal{S}}=\sum_{j \in \mathcal{S}} n_{j}$ denote the number of subdomain and point-wise measurements, respectively. It must be noted that matrix $\Pi$ is always invertible provided that the number of 


\begin{tabular}{|c|c|c|c|}
\hline & $\begin{array}{l}\text { Whole domain } \\
\begin{array}{c}\Omega=\underset{j \in \mathcal{J}}{\cup} \Omega_{j} \\
\mathcal{J}=1, \ldots, N\end{array}\end{array}$ & $\begin{array}{c}\text { Measurement domain } \\
\Omega_{\mathcal{S}}=\cup_{j \in \mathcal{S}} \Omega_{j} \\
\mathcal{S}=\mathcal{S}(1), \ldots, \mathcal{S}\left(N_{\mathcal{S}}\right)\end{array}$ & $\begin{array}{l}\text { Subdomains } \\
\qquad \Omega_{j}\end{array}$ \\
\hline Dimension & $n$ & $n_{\mathcal{S}}=\sum_{j \in \mathcal{S}} n_{j}$ & $n_{j}$ \\
\hline \multirow[t]{2}{*}{ Matrix $\mathcal{Z}$} & $\begin{array}{c}\mathcal{Z}=\left[\mathcal{Z}_{\mathcal{J}}(1)|\ldots| \mathcal{Z}_{\mathcal{J}}(m)\right] \\
\mathcal{Z} \in \mathbb{R}^{n \times m}\end{array}$ & $\begin{aligned} \mathcal{Z}_{\mathcal{S}}= & {\left[\mathcal{Z}_{\mathcal{S}}(1)|\ldots| \mathcal{Z}_{\mathcal{S}}(m)\right] } \\
& \mathcal{Z}_{\mathcal{S}} \in \mathbb{R}^{n_{\mathcal{S}} \times m}\end{aligned}$ & $\begin{array}{c}\mathcal{Z}_{j}=\left[\mathcal{Z}_{j}(1)|\ldots| \mathcal{Z}_{j}(m)\right] \\
\mathcal{Z}_{j} \in \mathbb{R}^{n_{j} \times m}\end{array}$ \\
\hline & $\mathcal{Z}_{\mathcal{J}}(i) \in \mathbb{R}^{n} \quad i=1, . ., m$ & $\mathcal{Z}_{\mathcal{S}}(i) \in \mathbb{R}^{n_{\mathcal{S}}} \quad i=1, \ldots, m$ & $\mathcal{Z}_{j}(i) \in \mathbb{R}^{n_{j}} \quad i=1, . ., m$ \\
\hline Matrix $\Pi$ & $\begin{array}{c}\Pi=\mathcal{Z}_{\mathcal{J}}^{T} \mathcal{Z}_{\mathcal{J}}=I \\
\Pi_{i, k}=\mathcal{Z}_{\mathcal{J}}(i)^{T} \mathcal{Z}_{\mathcal{J}}(k)=\delta_{i, k}\end{array}$ & $\begin{array}{c}\Pi=\mathcal{Z}_{\mathcal{S}}^{T} \mathcal{Z}_{\mathcal{S}} \\
\Pi_{i, k}=\mathcal{Z}_{\mathcal{S}}(i)^{T} \mathcal{Z}_{\mathcal{S}}(k)\end{array}$ & $\begin{array}{c}\Pi=\mathcal{Z}_{j}^{T} \mathcal{Z}_{j} \\
\Pi_{i, k}=\mathcal{Z}_{j}(i)^{T} \mathcal{Z}_{j}(k)\end{array}$ \\
\hline
\end{tabular}

Table 2: Summary of notation employed for the whole domain and subdomains of measurements

measurements $n_{\mathcal{S}}$ is larger than or equal to the dimension of the reduced space, i.e., that the rank of the $\mathcal{Z}_{\mathcal{S}}$ matrix is $m$. Under this assumption the vector of modes $\widehat{C}$ is identifiable (the solution is unique) since the least-squares function is quadratic on the modes with positive-definite Hessian.

The elements of the $\Pi$ matrix can be expressed in terms of the column vectors of the subdomain matrices $\left\{\mathcal{Z}_{j}\right\}_{j \in \mathcal{S}}$ :

$$
\Pi_{i, k}=\sum_{j \in \mathcal{S}} \mathcal{Z}_{j}(i)^{T} \mathcal{Z}_{j}(k) \equiv \mathcal{Z}_{\mathcal{S}}(i)^{T} \mathcal{Z}_{\mathcal{S}}(k) \quad \forall i, k=1, \ldots, m
$$

where the indexes in parenthesis indicate the positions of the columns inside the matrices $\mathcal{Z}_{j}$ and $\mathcal{Z}_{\mathcal{S}}$, respectively. Since, as we discussed previously, the POD set is orthonormal, we also have that:

$$
\lim _{\mathcal{S} \mapsto \mathcal{J}} \Pi_{i, k}=\delta_{i k} \quad \forall i, j
$$

with $\delta_{i k}$ being the Kronecker delta. In other words: as the subdomains of measurements cover the whole spatial domain, matrix $\Pi$ approaches the unity matrix. A summary of the notation employed is presented in Table 2. As we will show next, the proposed factorization and property (27) will turn out to be particularly convenient to the purpose of finding the optimal sensor locations for a given set of subdomains.

It must be pointed out that the proposed approach can be considered as an extension of previous works presented by Everson and Sirovich ${ }^{1}$ or Alonso et al. ${ }^{2,12}$. In fact, the methodology we develop can be applied not only to point-wise measurements, but also to measurements distributed over arbitrarily given spatial domains.

\subsection{Optimal sensor location}

The solution (25) suggests a criterion to locate sensors based on the degree of conditioning of matrix $\Pi$. In this way, given a number of sensors $N_{\mathcal{S}}$, the subdomains of measurement $\mathcal{S}$ will be chosen as those which maximize the minimum eigenvalue of $\Pi$. This is formally stated as:

$$
\max _{\mathcal{S}} \min _{i} \lambda_{i}(\Pi) \quad \text { with } \quad \Pi=\sum_{j \in \mathcal{S}} \mathcal{Z}_{j}^{T} \mathcal{Z}_{j}
$$


where $\lambda_{i}(\Pi)$ denotes the $i^{\text {th }}$ eigenvalue of $\Pi$. A geometric interpretation of such criterion has been proposed by Alonso et al. ${ }^{2}$ which connects the quality of the estimation with the angles between the low-dimensional and the measurement subspaces. These angles are, in fact, related to the eigenvalues $\lambda_{i}(\Pi)$ so that maximizing the minimum eigenvalue results into the minimization of the maximum angle between subspaces and thus the maximization of the estimation quality.

The dimension of the search space associated with problem (28) can be computed as a function of total number of subdomains $N$ and measurement subdomains $N_{\mathcal{S}}$, respectively, by the formula:

$$
d_{\mathcal{S}}=\frac{N !}{N_{\mathcal{S}} !\left(N-N_{\mathcal{S}}\right) !}
$$

This number becomes extraordinarily high even for a fairly limited number of subdomains what makes the use of exhaustive search to be usually prohibitive. In order to overcome such limitation, we follow the approach taken $i^{2,12}$ and approximate problem (28) by a much simpler one which consists of finding the set of measurement subdomains that maximizes the minimum diagonal element in $\Pi$. Formally, this criterion can be stated as:

$$
\max _{\mathcal{S}} \min _{i}\left(\Pi_{i, i}\right) \quad \text { with } \quad \Pi_{i, i}=\mathcal{Z}_{\mathcal{S}}(i)^{T} \mathcal{Z}_{\mathcal{S}}(i) \quad \forall i=1, \ldots, m
$$

where each vector $\mathcal{Z}_{\mathcal{S}}(i)$ is of the form:

$$
\mathcal{Z}_{\mathcal{S}}(i)=\left[\mathcal{Z}_{\mathcal{S}(1)}(i)^{T}, \ldots, \mathcal{Z}_{\mathcal{S}(j)}(i)^{T}, \ldots, \mathcal{Z}_{\mathcal{S}\left(N_{\mathcal{S}}\right)}(i)^{T}\right]^{T}
$$

As it was already pointed out by relation $(27)$, vectors $\mathcal{Z}_{\mathcal{S}}(i)$ become orthonormal as $\mathcal{S} \mapsto \mathcal{J}$. Thus solving problem (29) over the set $\mathcal{S}$ is nothing but looking for the nearest to orthonormal set of vectors $\left\{\mathcal{Z}_{\mathcal{S}}(i)\right\}_{i=1}^{m}$, which in addition will make $\Pi$ to be diagonal dominant. The Gershgorin disk theorem can then be employed to state the equivalence between problems (28) and (29) as discussed in ${ }^{2,12}$.

These arguments allow us to make use of the guided search algorithm developed by Alonso and coworkers details on the implementation can be found $\mathrm{in}^{2,12}$ ) for point-wise measurements, to optimal measurement subdomain location, i.e., to find those $N_{\mathcal{S}}$ subdomains among the total number $N$ which solve (29). A brief outline of the algorithm is presented in Appendix B.

\section{Case study: Rayleigh-Bénard problem}

In order to illustrate the approach discussed in the previous sections, we consider the problem of reconstructing the velocity field and temperature on a fluid under natural convection from a limited number of measurements. In particular, the case study corresponds with the $2 D$ version of the Rayleigh Bénard problem as described by Ly and Tran ${ }^{26}$. The spatial domain for the example is defined as:

$$
\Omega=\left\{\forall \xi_{1}, \xi_{2} \in \mathbb{R}:(0,1)\right\}
$$

with boundary $\partial \Omega=\Gamma_{1} \cup \Gamma_{2} \cup \Gamma_{3} \cup \Gamma_{4}$ :

$$
\begin{array}{ll}
\Gamma_{1}=\left\{\forall \xi_{2} \in \mathbb{R}:[0,1], \xi_{1}=1\right\} & \Gamma_{2}=\left\{\forall \xi_{1} \in \mathbb{R}:[0,1], \xi_{2}=1\right\} \\
\Gamma_{3}=\left\{\forall \xi_{2} \in \mathbb{R}:[0,1], \xi_{1}=0\right\} & \Gamma_{4}=\left\{\forall \xi_{1} \in \mathbb{R}:[0,1], \xi_{2}=0\right\}
\end{array}
$$

The dimensionless PDEs for the velocity $\vec{v}=\left[v_{x}, v_{y}\right]^{T}$ and the temperature $T$ are:

$$
\gamma \frac{\partial \vec{v}}{\partial t}+\gamma \vec{v} \nabla \vec{v}=-\nabla P+\gamma \vec{j} T+\Delta \vec{v}
$$




$$
\begin{gathered}
\gamma \frac{\partial T}{\partial t}+\gamma \vec{v} \nabla T=\Delta T \\
\nabla \vec{v}=0
\end{gathered}
$$

being $\vec{j}$ the vertical unitary vector and denoting by $\gamma$ a dimensionless parameter that measures the relation between the Rayleigh and Prandt numbers. The model is completed with the following boundary and initial conditions:

$$
\begin{gathered}
v_{x}(\xi, t)=v_{y}(\xi, t)=0, \quad \frac{\partial T(\xi, t)}{\partial \vec{n}}=0 \quad \forall \xi \in \Gamma_{2} \cup \Gamma_{4}, \forall t \in \mathbb{R}^{+} \\
v_{x}(\xi, t)=v_{y}(\xi, t)=0, \quad T(\xi, t)=0 \quad \forall \xi \in \Gamma_{3}, \forall t \in \mathbb{R}^{+} \\
v_{x}(\xi, t)=v_{y}(\xi, t)=0, \quad T(\xi, t)=1 \quad \forall \xi \in \Gamma_{1}, \forall t \in \mathbb{R}^{+} \\
\vec{v}(\xi, t)=0 \quad \forall \xi \in \Omega, t=0 \\
T(\xi, t)=0 \quad \forall \xi \in \Omega, t=0
\end{gathered}
$$

The experiments were carried out in $\mathrm{FEMLAB}^{27}$ with a discretization of $\mathbf{n}=1681$ points (further refinements in the mesh resulted into negligible improvements on the accuracy of the results) and $\gamma=500$, so that, relevant natural convection behavior is shown.

The basis of the low-dimensional subspace, associated with the discrete two-point correlation kernel (20), were obtained from a large enough number of such snapshots $(\ell=1815)$ taken each 0.2 dimensionless units of time and ordered sequentially in a vector $T \in \mathbb{R}^{\mathbf{n}}$ for the temperature and $V=\left[V_{x}^{T} V_{y}^{T}\right]^{T} \in \mathbb{R}^{2 \mathbf{n} \times \ell}$ for the velocity. Figure 2 shows the energy captured by the first 40 PODs according to criterion (17). We consider the first 23 temperature and 39 velocity PODs to be representative of the relevant dynamics of the system since they are able to capture the $99.99 \%$ of the total energy . Note the reduction of two orders of magnitude when working with the global basis set (estimation requires of 62 modes $\widehat{C}$ ) instead of a local basis approach (the dimension is now $\mathbf{n}=1681$ for each field and 5043 modes are needed).

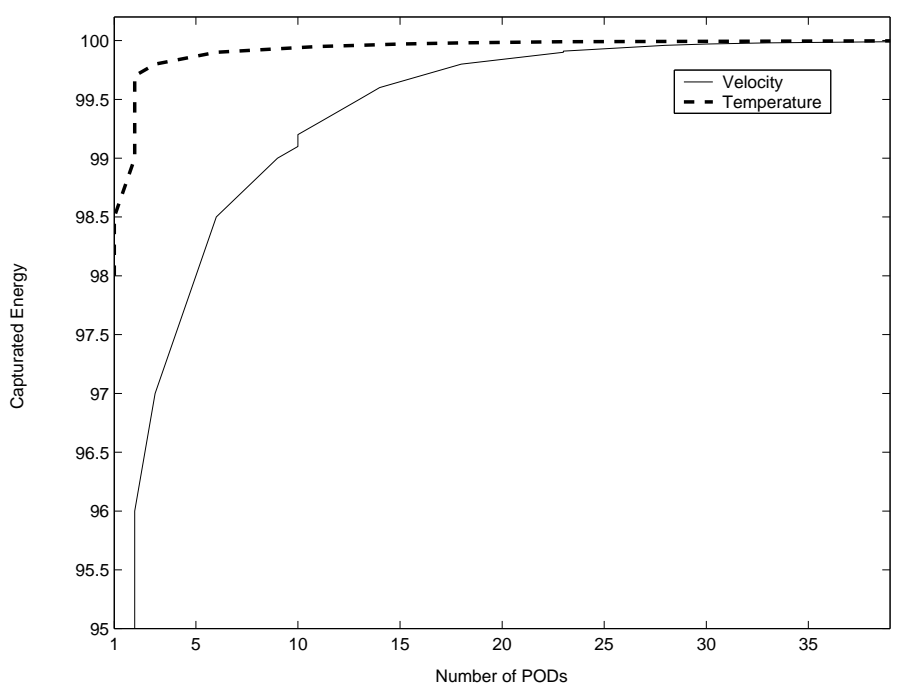

Figure 2: Energy captured as a function of the number of PODs for the temperature and the velocity fields

In order to illustrate the time scale separation property of the PODs, the time dependent functions $\widehat{C}$ were obtained at each sampling time $t_{s}$ by using the expression (25) extended over the whole domain $\Omega$. Figure 3 shows the five dominant modes where, in fact, we see the amplitude 


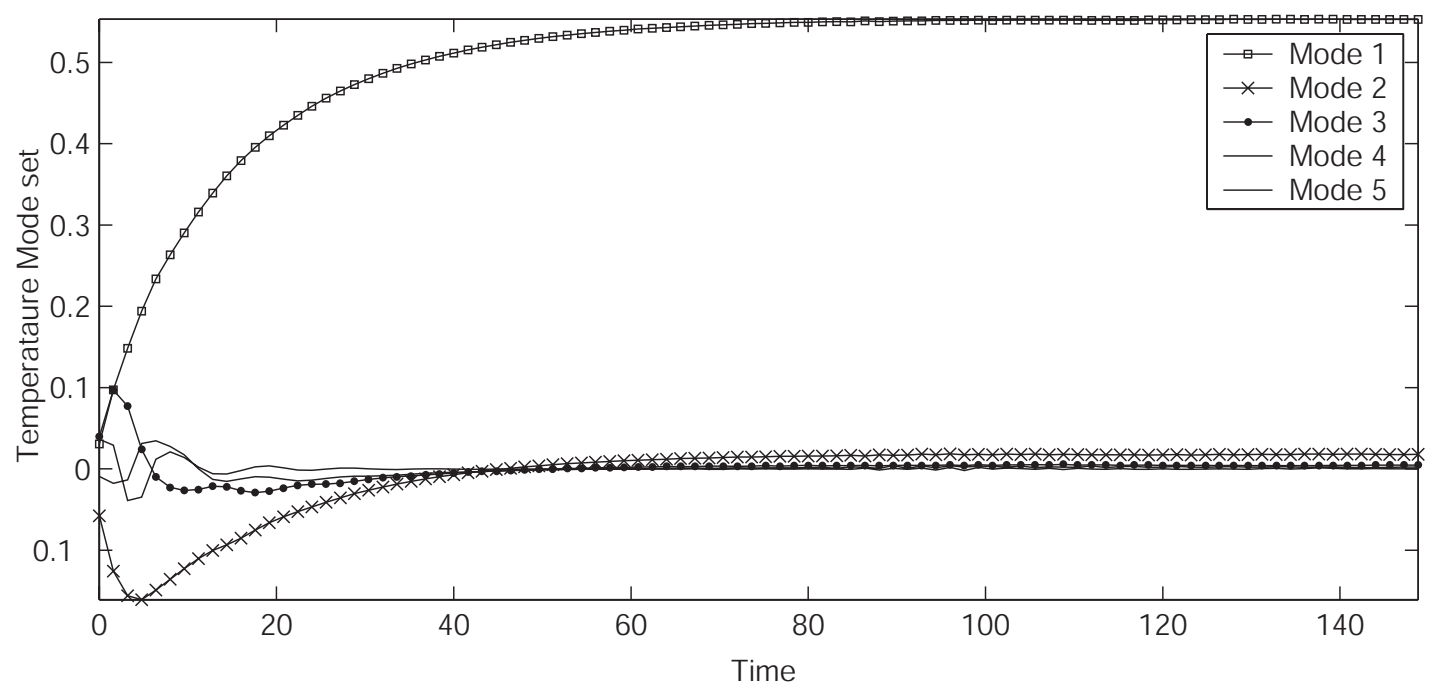

Figure 3: Evolution of the first (dominant) five modes of the temperature snapshots

differences among them and the ability of the first terms of the temperature expansion (15) to reproduce the most relevant dynamics.

The optimal sensor location problem is formulated as in section 4.2 and consists of finding the best possible 5 subdomains of measurement (each of dimensions $0.2 \times 0.2$ length units) among 25 subdomains of equal size covering the whole domain $\Omega$. The algorithm developed $\mathrm{in}^{2,12}$ was used to find the optimal locations among the 53130 combinations, according to criterion (29). Optimal placements for temperature and velocity are presented in Figures $4 \mathrm{a}$ and $4 \mathrm{~b}$, respectively. In order

a)

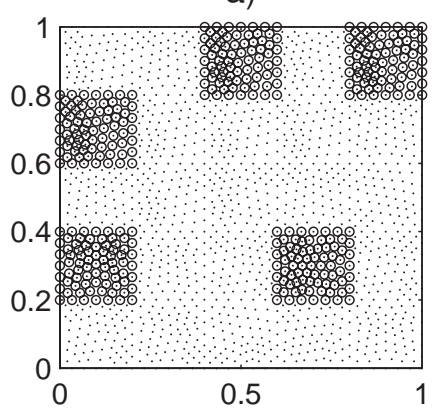

b)

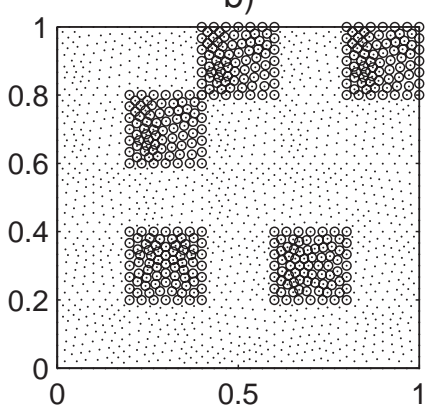

c)

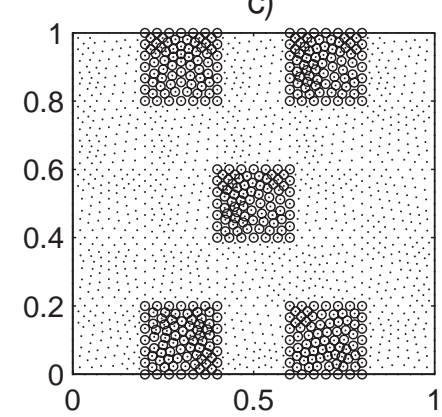

Figure 4: Optimal sensor location for a) Temperature and b) Velocity and c) a suboptimal scenario for temperature

to illustrate the dependence of sensor arrangements on the quality of the estimation, we compare the optimal placements with a suboptimal, although a priori acceptable, scenario depicted in Figure $4 \mathrm{c}$ and chosen by heuristic arguments (the sensors are not clustered and are located in subdomains where relevant dynamics take place).

The estimated temperature evolution in terms of the first two dominant modes for both arrangements is illustrated in Figure 5. As it can be observed in the figure, the modes estimated from suboptimal sensor placement present anomalous oscillations as compared with the optimal placement, which display a behavior almost identical to the real modes computed directly from the snapshots.

The differences in the quality of the reconstruction obtained from these arrangements can also be observed in Figure 6 where Figure 6a represents a given snapshot and Figures $6 \mathrm{~b}$ and $6 \mathrm{c}$ the reconstruction obtained from the optimal and suboptimal arrangements, respectively. While the 


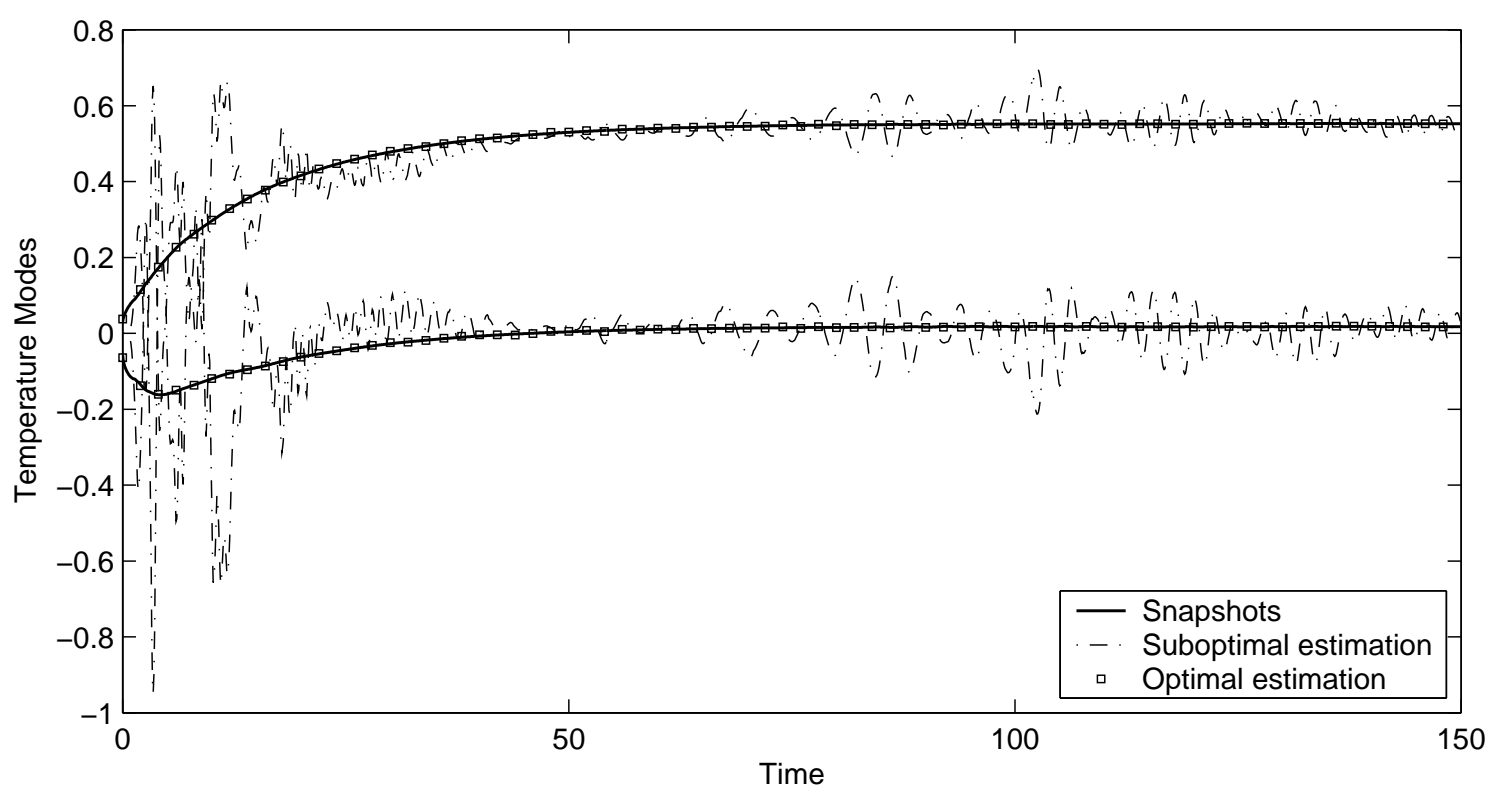

Figure 5: Evolution of the dominant temperature modes obtained from snapshots (line) and their corresponding estimations using the optimal (squares) and suboptimal (dash-dotted line) placement scenarios

optimal placement is able to reproduce almost exactly the real behavior, the suboptimal configuration results into a poor approximation (specially in reproducing the temperature distribution near the right boundary region). The same trend has been observed for the entire transients in temperature as well as in the reconstruction of the velocity field distribution (see Figure 7) from the optimal arrangement depicted in Figure $4 \mathrm{~b}$.
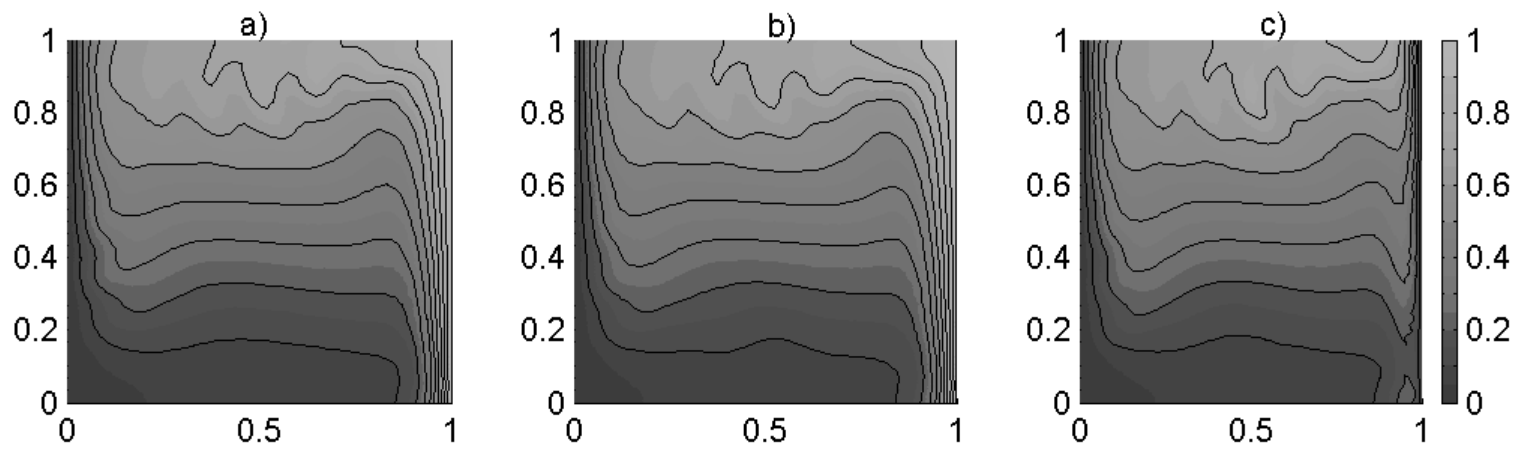

Figure 6: Temperature spatial distribution at 25 units of time: (a) snapshot obtained from direct numerical simulation, (b) reconstruction from optimal sensor placement and (c) reconstruction from suboptimal sensor placement 

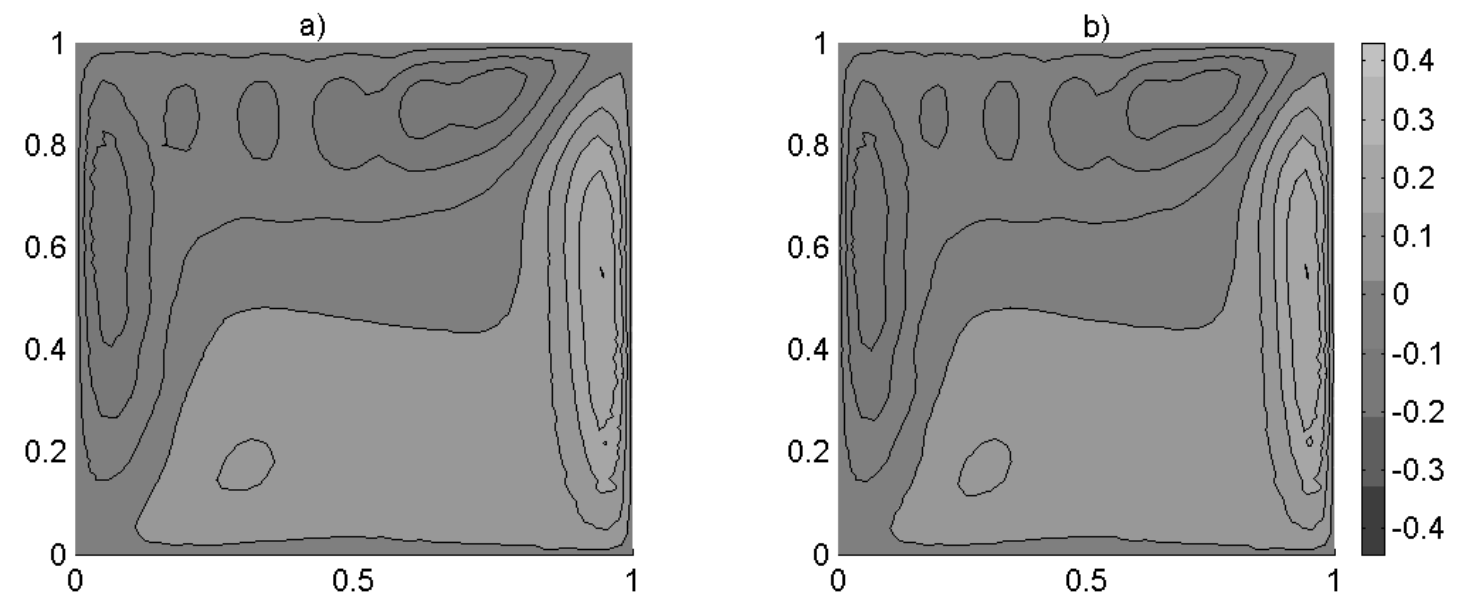

Figure 7: Spatial distribution of the velocity field $\left(v_{x}+v_{y}\right)$ at 25 units of time: (a) snapshot obtained from direct numerical simulation and (b) reconstruction from optimal sensor placement

\section{Conclusions}

In this work, a systematic framework for efficient field reconstruction of nonlinear distributed process systems in arbitrary geometries has been proposed. The approach is of particular interest in dealing with complex nonlinear phenomena such as that exhibited in fluid dynamics or nonlinear diffusion-reaction systems. In this context, reconstruction usually requires of reduced order representations of the field as well as large sets of sensors to be placed on the domain in order to capture the representative dynamic features of the field.

The methodology takes advantage of the underlying algebraic structure of the FEM framework and combines it with previous results in optimal sensor location to develop field reconstructions in terms of globally defined basis functions and to select optimal placement of sensors based on the concept of measurement subdomain. At this point it must be pointed out that the FEM framework can be further exploited to derive efficient low dimensional dynamic approximations in arbitrary $2 D$ or $3 D$ geometries thus extending reconstruction capabilities. The ideas proposed in this contribution have been illustrated on reconstructing temperature and velocity fields on a fluid under natural convection.

\section{Acknowledgments}

The authors acknowledge financial support received from the Spanish Government (DPI2004-07444C04-03) and Xunta de Galicia (PGIDIT02-PXIC40209PN). 


\section{References}

(1) Everson, R.; Sirovich, L. Karhunen-loeve procedure for gappy data. Journal of the Optical Society of America, 1995, 12(8), 1657.

(2) Alonso, A. A.; Frouzakis, C. E.; Kevrekidis, I. G. Optimal sensor placement for state reconstruction of distributed process systems. AIChE Journal, 2004, 50(7), 1438.

(3) Cohen, K.; Siegel, S.; McLaughlin, T. A heuristic approach to effective sensor placement for modeling of a cylinder wake. Comput. Fluids, 2006, 35(1), 103.

(4) Kumar, S. and Seinfeld, J. Optimal location of measurements for distributed parameter estimation. IEEE Trans. Autom. Control, 1978, 23, 690.

(5) Alvarez, J.; Romagnoli, J.; Stephanopoulos, G. Variable measurement structures for the control of a tubular reactor. Chem. Eng. Sci., 1981, 36(10), 1695.

(6) Harris, T.; Wright, J.; MacGregor, J. Optimal sensor location with an application to a packed bed tubular reactor. AIChE Journal, 1980, 26(6), 910.

(7) Vande Wouwer, A.; Point, N.; Porteman, S.; Remy, M. An approach to the selection of optimal sensor locations in distributed parameter systems. Journal of Process Control, 2000, 10(4), 291.

(8) Van den Berg, F.W.J.; Hoefsloot, H.C.J.; Boelens, H.F.M.; Smilde, A.K. Selection of optimal sensor position in a tubular reactor using robust degree of observability criteria. Chemical Engineering Science, 2000, 55, 827.

(9) Kubrusly, C. and Malebranche, H. Sensors and controllers location in distributed systems-a survey. Automatica, 1992, 21(2), 117.

(10) Antoniades, C. and Christofides, P. D. Integrating nonlinear output feedback control and optimal actuator/sensor placement for transport-reaction processes. Chemical Engineering Science, 2001, 56(15), 4517.

(11) Demetriou, M. and Kazantzis, N. A new integrated output feedback controller synthesis and collocated actuator/sensor scheduling framework for distributed parameter processes. Comput. Chem. Eng., 2005, 29(4), 867.

(12) Alonso, A. A.; Kevrekidis, I. G.; Banga, J. R.; Frouzakis, C. E. Optimal sensor location and reduced order observer design for distributed process systems. Computers and Chemical Engineering, 2004, 28(1-2), 27.

(13) Christofides, P. D. Nonlinear and Robust Control of PDE Systems: Methods and Applications to Transport-Reaction Processes. Birkhäuser, Boston, 2001.

(14) Alonso, A. A.; Banga, J. R.; Sánchez, I. Passive control design for distributed process systems: Theory and applications. AIChE Journal, 2000, 46(8), 1593.

(15) Alonso, A. A. and Ydstie, B. E. Stabilization of distributed systems using irreversible thermodynamics. Automatica, 2001, 37(11), 1739. 
(16) Alonso, A. A.; Fernández, C. V.; Banga, J. R. Dissipative systems: from physics to robust nonlinear control. Int. J. Robust Nonlinear Control, 2004, 14(2), 157.

(17) Sirovich, L. Turbulence and the dynamics of coherent structures. Part I: Coherent structures. Quaterly of Appl. Math., 1987, 45(3), 561.

(18) Berkooz, G.; Holmes, P.; Lumley, L. The Proper Orthogonal Decomposition in the analysis of turbulent flows. Ann. Rev. Fluid Mech., 1993, 25, 539.

(19) Walter, E. and Pronzato, L. Identification of Parametric Models. Springer, Great Britain, 1st edition, 1994.

(20) Willcox, K. Unsteady flow sensing and estimation via the gappy proper orthogonal decomposition. Computers \& fluids, 2006, 35(2), 208.

(21) Courant, R. and Hilbert, D. Methods of Mathematical Physics. John Wiley \& Sons, New York, USA, 1st edition, 1989.

(22) Reddy, J. N.An Introduction to the Finite Element Method. McGraw-Hill, 2nd edition, 1993.

(23) Ravindran, S. S. A reduced-order approach for optimal control of fluids using proper orthogonal decomposition. International journal for numerical methods in fluids, 2000, $34(5), 425$.

(24) Cristianini, N. and Shawe-Taylor, J.An Introduction to Support Vector Machines. Cambridge University Press, Cambridge, 2000.

(25) Golub, G. and Van Loan, C. Matrix Computations. John Hopkins University Press, 1996.

(26) Ly, H. V. and Tran, H. T. Modeling and control of physical processes using proper orthogonal decomposition. Mathematical and Computer Modelling ,2001, 33(1-3), 223.

(27) COMSOL AB. FEMLAB reference manual version 2.0. Tegnergatan 23, SE-111 40 Stockholm, Sweden, 2000. 


\section{Appendix A}

Let $f, h \in H^{1}(\Omega)$ be two arbitrary functions whose expansion in the FEM basis are:

$$
\begin{aligned}
& f(\xi) \simeq \sum_{\mathbf{i}=\mathbf{1}}^{\mathbf{n}} \mathrm{f}_{\mathbf{i}} \psi_{\mathbf{i}}(\xi)=\bar{f}^{T} \bar{\psi}(\xi) \\
& h(\xi) \simeq \sum_{\mathbf{i}=\mathbf{1}}^{\mathbf{n}} \mathrm{h}_{\mathbf{i}} \psi_{\mathbf{i}}(\xi)=\bar{h}^{T} \bar{\psi}(\xi)
\end{aligned}
$$

with coordinate vectors $\bar{f} \in \mathbb{R}^{\mathbf{n}}$ and $\bar{h} \in \mathbb{R}^{\mathbf{n}}$ and the FEM basis vector $\bar{\psi}(\xi) \in \mathbb{R}^{\mathbf{n}}$ ordered as:

$$
\bar{\psi}(\xi)=\left[\psi_{\mathbf{1}}(\xi), \ldots, \psi_{\mathbf{i}}(\xi), \ldots, \psi_{\mathbf{n}}(\xi)\right]^{T} \quad \bar{f}=\left[f_{\mathbf{1}}, \ldots, f_{\mathbf{i}}, \ldots f_{\mathbf{n}}\right]^{T} \quad \bar{h}=\left[h_{\mathbf{1}}, \ldots, h_{\mathbf{i}}, \ldots h_{\mathbf{n}}\right]^{T}
$$

The following correspondences between the infinite-dimensional space $H^{1}(\Omega)$ and the finite one $X_{\mathbf{n}}$ can be established:

\section{Spatial integration.}

$$
\int_{\Omega} h(\xi) f(\xi) d \xi=\int_{\Omega}\left(\bar{h}^{T} \bar{\psi}(\xi) \bar{\psi}(\xi)^{T} \bar{f}\right) d \xi=\bar{h}^{T}\left(\int_{\Omega} \bar{\psi}(\xi) \bar{\psi}(\xi)^{T} d \xi\right) \bar{f}
$$

As it was discussed in the first Section, the expansion coefficients coincide with the value of the variables in the mesh nodes (7). Hence, using the FEM matrices defined in (10) the spatial integration can be numerically approximated as:

$$
\int_{\Omega} h(\xi) f(\xi) d \xi \longrightarrow H^{T}(\mathcal{D} \mathcal{A}) F
$$

Spatial integration of the gradient of an arbitrary function. By analogy with the former case:

$$
\int_{\Omega} h(\xi) \nabla f(\xi) d \xi=\bar{h}^{T}\left(\int_{\Omega} \bar{\psi}(\xi) \nabla \bar{\psi}(\xi)^{T} d \xi\right) \bar{f} \longrightarrow H^{T}(\mathcal{B E}) F
$$

Spatial integration of the Laplacian of an arbitrary function. Using the divergence theorem we have:

$$
\int_{\Omega} h(\xi) \triangle f(\xi) d \xi=\int_{\partial \Omega} h \vec{n} \nabla f d \xi-\int_{\Omega} \nabla h \nabla f d \xi
$$

inserting boundary condition $\vec{n} \nabla f+q f=g$ into equation (36) leads to:

$$
\begin{gathered}
\int_{\partial \Omega} h \vec{n} \nabla f d \xi-\int_{\Omega} \nabla h \nabla f d \xi=\int_{\partial \Omega} h g d \xi-q \int_{\partial \Omega} h f d \xi-\int_{\Omega} \nabla h \nabla f d \xi= \\
=\bar{h}^{T}\left(\int_{\partial \Omega} \bar{\psi} \bar{g}^{T} d \xi\right)-\bar{h}^{T} q\left(\int_{\partial \Omega} \overline{\psi \psi}^{T} d \xi\right) \bar{f}-\bar{h}^{T}\left(\int_{\partial \Omega} \nabla \bar{\psi} \nabla \bar{\psi}^{T} d \xi\right) \bar{f}
\end{gathered}
$$

and the mapping between the continuous and discrete version becomes:

$$
\int_{\Omega} h(\xi) \triangle f(\xi) d \xi \longrightarrow H^{T} \mathcal{G}-H^{T}(q \mathcal{Q}+\mathcal{C}) F
$$


Note that all the relationships shown here, consist of integrations over the spatial domain. The implementations of the Laplacian or gradient, however, can be easily extracted using also these mappings. For instance, let us denote as $\nabla F$ the discrete gradient version of the function $f(\xi)$ :

$$
\int_{\Omega} h(\xi) \nabla f(\xi) d \xi \longrightarrow H^{T}(\mathcal{D} \mathcal{A}) \nabla F=H^{T}(\mathcal{B E}) F
$$

since this equality holds for every function $h(\xi) \in H^{1}(\Omega)$ we can conclude that:

$$
\begin{gathered}
(\mathcal{D} \mathcal{A}) \nabla F=(\mathcal{B E}) F \Rightarrow \nabla F=\mathcal{D} \mathcal{A}^{-1}(\mathcal{B E}) F \\
\nabla f(\xi) \longrightarrow \mathcal{D} \mathcal{A}^{-1}(\mathcal{B E}) F
\end{gathered}
$$

The same arguments can be applied to the Laplacian operator. 


\section{Appendix B}

Firstly, we define a set of $\mathrm{m}$ N-dimensional vectors $\varrho_{i}$ with elements $\varrho_{i}=\left[\mathcal{Z}_{1}(i)^{T} \mathcal{Z}_{1}(i), \ldots, \mathcal{Z}_{N}(i)^{T} \mathcal{Z}_{N}(i)\right]$ and associate with each of them an index vector set $\left\{\eta_{i}^{(p)}\right\}$ with $\eta_{i}^{(p)} \in \mathbb{R}^{N_{\mathcal{S}}}$ and an operator $\Im\left[\varrho_{j} ;\left\{\eta_{i}^{(p)}\right\}\right]$ which computes all summations of the $\left\{\eta_{i}^{(p)}\right\}$ elements of $\varrho_{j}$. Search will proceed on a m-dimensional space of all possible $N_{\mathcal{S}}$-summations by constructing k-sequences of $\left\{\eta_{i}^{(p)}\right\}^{k}$ for each $i=1, . ., m$ ordered so that $\Im\left[\varrho_{j} ;\left\{\eta_{i}^{(p)}\right\}\right] \leq \Im\left[\varrho_{j} ;\left\{\eta_{i}^{(p-1)}\right\}\right]$. At iteration 1 , we compute $\left\{\eta_{i}^{(p)}\right\}^{1}$ for $i=1, . ., m$, their corresponding summations $\Im\left[\varrho_{j} ;\left\{\eta_{i}^{(p)}\right\}^{1}\right]$ and the value $L^{(1)}=\max _{i}\left(\Im\left[\varrho_{j} ;\left\{\eta_{i}^{(1)}\right\}\right]\right)$. This value is an approximation to the solution of problem (29) and will be used in the next iteration to select only those sequence elements that satisfy $L^{(2)} \geq L^{(1)}$. The procedure is then repeated until no sequence, improving the previous best max-min value, exist. This is illustrated in Figure 8 for $m=2$ and $N_{\mathcal{S}}=3$ and where dots indicate possible solutions. After the first iteration $L^{(1)}$ determines the bounds (shadowed rectangle) where a better solution can not be found. This step reduces the number of sequence elements to be computed in the next iteration. In fact after the first iteration $\left\{\eta_{1}^{(2)}\right\}$ is the only sequence element that could improve $L^{(1)}$ that coincides with the best previous vale (for more details $\operatorname{see}^{2,12}$ ).

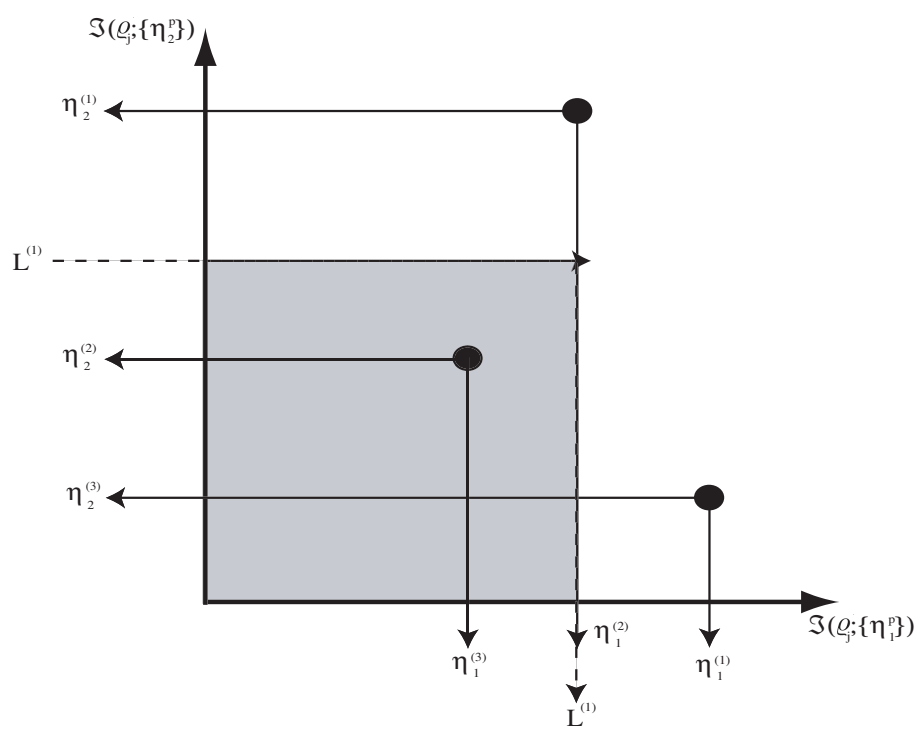

Figure 8: Structure of the search space 


\section{List of Figures}

1 The spatial subdomain $\Omega$ is decomposed into 13 non-overlapping subdomains $\Omega_{j}$ with $\mathcal{J}=(1, \ldots, 13)$. Highlighted in grey is a possible set of measurement subdomains

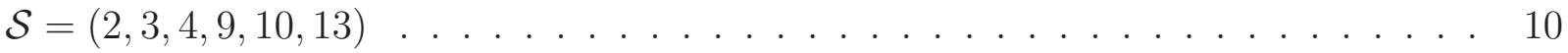

2 Energy captured as a function of the number of PODs for the temperature and the velocity fields . . . . . . . . . . . . . . . . . . . . . 13

3 Evolution of the first (dominant) five modes of the temperature snapshots . . . . . . 14

4 Optimal sensor location for a) Temperature and b) Velocity and c) a suboptimal scenario for temperature . . . . . . . . . . . . . . . . . . . . 14

5 Evolution of the dominant temperature modes obtained from snapshots (line) and their corresponding estimations using the optimal (squares) and suboptimal (dashdotted line) placement scenarios . . . . . . . . . . . . . . . . . 15

6 Temperature spatial distribution at 25 units of time: (a) snapshot obtained from direct numerical simulation, (b) reconstruction from optimal sensor placement and (c) reconstruction from suboptimal sensor placement . . . . . . . . . . . 15

7 Spatial distribution of the velocity field $\left(v_{x}+v_{y}\right)$ at 25 units of time: (a) snapshot obtained from direct numerical simulation and (b) reconstruction from optimal sensor placement . . . . . . . . . . . . . . . . . . . . 16

8 Structure of the search space . . . . . . . . . . . . . . . . . . 21 